\title{
Effects of metallothionein-3 and metallothionein-1E gene transfection on proliferation, cell cycle, and apoptosis of esophageal cancer cells
}

\author{
Z.Q. Tian', Y.Z. Xu' ${ }^{1}$, Y.F. Zhang ${ }^{1}$, G.F. $\mathrm{Ma}^{1}$, M. $\mathrm{He}^{1}$ and G.-Y. Wang ${ }^{2}$ \\ ${ }^{1}$ Department of Thoracic Surgery, \\ The Fourth Hospital of Hebei Medical University, Shijiazhuang, \\ Hebei Province, China \\ ${ }^{2}$ The Second Department of General Surgery, \\ The Fourth Hospital of Hebei Medical University, Shijiazhuang, \\ Hebei Province, China \\ Corresponding author: G.-Y. Wang \\ E-mail: tizq_12@yeah.net
}

Genet. Mol. Res. 12 (4): 4595-4603 (2013)

Received February 5, 2013

Accepted July 18, 2013

Published October 17, 2013

DOI http://dx.doi.org/10.4238/2013.October.17.2

\begin{abstract}
Metallothionein (MT)-3 has cell growth inhibitory activity, and is the only currently known MT subtype with unique physiological functions. The expression levels of MT-1E, a subtype of MT-1, were positively correlated with the degree of esophageal cancer malignancy. The present study aimed to investigate the effects of MT-3 and MT-1E gene transfection on the proliferation, cell cycle, and apoptosis of esophageal cancer cells. The cationic liposome method was used to transfect the esophageal cancer strains Eca-109 and TE13. Reverse transcription-polymerase chain reaction was used to detect target gene expression, 3-(4,5-dimethylthiazol-2-yl)-2,5-diphenyltetrazolium bromide reduction was applied to detect cell proliferation, and flow cytometry was used for cell cycle and apoptosis detection. Esophageal
\end{abstract}


cancer cells with MT-3 and MT-1E gene transfection showed high expression of the foreign target gene and mRNA. Cells with MT-3 gene transfection showed markedly inhibited proliferation $(\mathrm{P}<0.05)$, a significantly higher proportion of cells in the $\mathrm{G} 0 / \mathrm{G} 1$ phase $(\mathrm{P}<0.05)$, a significantly lower proportion of cells in the $\mathrm{S}$ phase $(\mathrm{P}<0.05)$, and a significantly increased apoptosis rate $(\mathrm{P}<0.05)$. Cells with MT-1E gene transfection did not show significant changes in proliferation, cell cycle, or apoptosis rate $(\mathrm{P}>0.05)$. Therefore, the upregulation of MT-3 gene expression can inhibit esophageal cancer cell proliferation and induce apoptosis, which may be achieved by blocking the tumor cell growth cycle, whereas effects of the MT-1E gene on the proliferation of esophageal cancer cells were not evident.

Key words: MT-3; MT-1E; Esophageal cancer; Gene transfection; Flow cytometry; Proliferation

\section{INTRODUCTION}

Metallothionein (MT) is a low molecular weight and cysteine-rich protein that is widely distributed in organisms in vivo. Four isomers have been found to date in the MT family, named MT-1 to MT-4. MT-1 and MT-2 expressions have been widely studied with respect to esophageal cancer. Hishikawa et al. (1999) found that the high level of MT expression in esophageal cancer (mainly MT-1 and MT-2) was associated with lymph node metastasis, distant metastasis, and poor prognosis. Sun et al. (2006) also found that high expressions of MT-1 and MT-2 were closely associated with the progress, low degree of differentiation, and lymph node metastasis of the esophageal disease. The MT-3 gene is a novel gene whose importance has only been noted in recent years. MT-3 gene hypermethylation was demonstrated in esophageal carcinoma, and this hypermethylation may lead to a reduction in MT-3 expression levels (Tian and Liu, 2004; Smith et al., 2005). In this study, using gene transfection technology, we artificially increased the expression levels of the MT-3 and MT-1E genes in esophageal cancer cells. Subsequently, proliferation, cell cycle, and apoptosis changes were detected in esophageal carcinoma cells. This study was designed to further clarify the roles that different subtypes of MT might play in esophageal cancer.

\section{MATERIAL AND METHODS}

\section{Main materials}

The human esophageal carcinoma Eca-109 and TE13 cell strains were obtained from the Scientific Research Center of the Fourth Hospital of Hebei Medical University. Four plasmids were designed and synthesized by the Guangzhou Funeng Genes Co., Ltd. The plasmid containing the MT-3 gene was EX-T3737-M03, and its corresponding empty vector was EX-NEG-M03; these two plasmids also contained the green fluorescent protein gene. Lipofectamine $^{\mathrm{TM}} 2000$ was purchased from the Invitrogen Corporation (USA). Four methyl thiazolyl tetrazolium (MTT), dimethyl sulfoxide (DMSO), propidium iodide (PI), and RNase A were all purchased from Sigma (USA). The two-step reverse transcription-polymerase chain 
reaction (RT-PCR) kit was purchased from Promega Corporation (USA). The RPMI 1640 powder was purchased from Gibco (USA).

\section{Cell culture}

The human esophageal cancer cell strains Eca-109 and TE13 were cultured in RPMI 1640 medium containing $10 \%$ fetal bovine serum, and were placed in a routinely humidified incubator at $37^{\circ} \mathrm{C}$ and $5 \% \mathrm{CO}_{2}$ for culturing. The logarithmic growth phase cells were selected for subsequent experiments.

\section{Gene transfection}

Eca-109 and TE13 cells were seeded on 6-well plates at a density of 2-3 x $10^{5}$ cells/ well, and cultured overnight. When cell growth reached approximately $70 \%$, the transfection was initiated according to Lipofectamine ${ }^{\mathrm{TM}} 2000$ reagent instructions. At 24, 48, and $72 \mathrm{~h}$ after transfection, fluorescence microscopy was used to observe the fluorescent protein expression in the plasmids of cells in order to determine the transfection efficiency. Cells were divided into five groups: the EX-T3737-M03-transfected group, the EX-NEG-M03-transfected group, the EX-H2598-M56-transfected group, the EX-NEG-M56-transfected group, and the blank control group. Three replicate wells were established in each group.

\section{MT-3 and MT-1E gene expression after RT-PCR detection and transfection in cells of each group}

Each transfection group and the blank control group were cultured for $48 \mathrm{~h}$, at which point the cells were collected. The RT-PCR was performed as described in Guo and Tian (2010) using the following primers: upstream primer of the amplified MT-3 gene (5'-GACCTGC CCCTGCCCTTCTGGTGG-3') and the reverse primer (5'-GCTCCACACGGAGGGGTGCC TTCT-3'), with a fragment size of $217 \mathrm{bp}$; MT-1E gene upstream primer (5'-GCTTGTTCGTC TCACTGGTG-3') and reverse primer (5'-CAGGTTGTGCAGGTTGTTCTA-3'), fragment size of $284 \mathrm{bp}$; GAPDH (internal reference) gene upstream primer (5'-AGGTGAAGGTCGGA GTCAACG-3') and reverse primer (5'-AGGGGTCATTGATGGCAACA-3'), fragment size of $104 \mathrm{bp}$. The PCR products were loaded on $1.5 \%$ agarose gel for electrophoresis. The AlphaInnotech FluorChem 8900 fluorescent gel imaging chemiluminescence system was used to analyze the results.

\section{MTT assay to detect cell proliferation ability}

Cells $\left(1 \times 10^{4}\right.$ cells/well) were seeded on a 96-well cell culture plate, in which the volume of each well was $100 \mu \mathrm{L}$. Six wells were established for each group, and blank wells were also set. After overnight incubation and transfection (as described above), $20 \mu \mathrm{L} 5 \mathrm{mg} / \mathrm{mL}$ MTT solution was added to each well at 24,48 , and $72 \mathrm{~h}$, respectively. Cells were cultured for $4 \mathrm{~h}$ at $37^{\circ} \mathrm{C}$, the culture medium was removed, and $150 \mu \mathrm{L}$ DMSO was added in each well. After shaking for $10 \mathrm{~min}$, the absorbance $(D)$ values at a $492 \mathrm{~nm}$ wavelength were detected for each group. The experiment was repeated three times. The average $D$ value was plotted on the longi- 
tudinal axis, and the incubation time was plotted on the horizontal axis to draw the cell growth curve. The proliferation and inhibition rate of cells was calculated according to the formula: cell proliferation and inhibition rate $=\left(1-D_{\text {mean in transfected group }} / D_{\text {mean in blank control group }}\right) \times 100 \%$.

\section{Flow cytometry for the cell cycle and apoptosis detection}

All transfection groups and the blank control group were cultured for $48 \mathrm{~h}$, the cells were digested, collected, washed with cold phosphate-buffered saline twice, and made into single cell suspensions. Suspensions were immediately added to precooled $70 \%$ ethanol at $4{ }^{\circ} \mathrm{C}$ for fixation, centrifuged at $1000 \mathrm{rpm}$ for $5 \mathrm{~min}$, and the supernatant was removed. Cell suspensions were then filtered through a 300-mesh sieve, placed into the RNA enzyme digestion for $30 \mathrm{~min}, 1 \mathrm{~mL} 0.05 \mathrm{~g} / \mathrm{L}$ PI was added, the cell density was adjusted to $1 \times 10^{6}$ cells/L, and they were stained at $4^{\circ} \mathrm{C}$ in the dark for $30 \mathrm{~min}$. Flow cytometry analysis was conducted on these samples at an excitation wavelength of $488 \mathrm{~nm}$. This entire process was repeated three times.

\section{Statistical analysis}

Data are reported as means \pm SD. The SPSS11.5 statistical software was used to perform the Student $t$-test, and $\mathrm{P}<0.05$ was considered as a statistically significant difference.

\section{RESULTS}

\section{Transfection efficiency based on fluorescence microscopy}

Fluorescence microscope observations revealed fluorescent protein expressions at 24, 48, and $72 \mathrm{~h}$ after Eca-109 and TE13 cell strain transfections in the EX-T3737-M03-, EXNEG-M03-, EX-H2598-M56-, and EX-NEG-M56-transfected groups. The cell fluorescence expression intensity was strongest at $48 \mathrm{~h}$ after transfection, representing approximately $90 \%$ of the total number of cells (Figure 1).

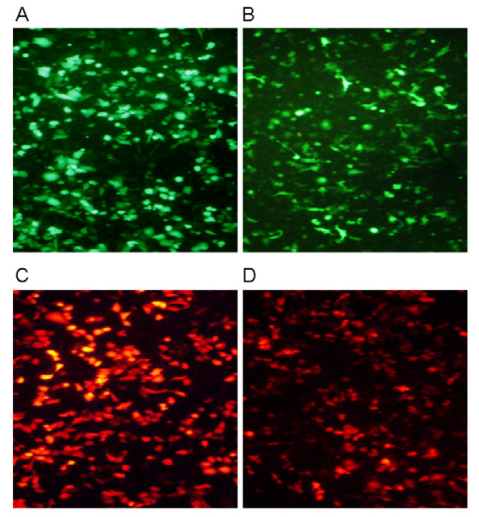

Figure 1. Expression of fluorescent protein in transfected esophageal cancer cells through fluorescence microsope after transfection for $48 \mathrm{~h}$ (100X). A. Transfection with EX-T3737-M03 in Eca-109 cells. B. Transfection with EX-T3737-M03 in TE13 cells. C. Transfection with EX-H2598-M56 in Eca-109 cells. D. Transfection with EX-H2598-M56 in TE13 cells. 


\section{MT-3 and MT-1E gene and mRNA expression levels after transfection}

The semi-quantitative RT-PCR results showed that in contrast to the EX-T3737-M03and EX-NEG-M03-transfected groups, MT-3 gene and mRNA expression levels of the blank group were significantly increased in the Eca-109 and TE13 cell strains $(\mathrm{P}<0.05)$. MT-3 gene and mRNA expression levels did not differ significantly between the EX-NEG-M03-transfected group and the blank control group $(\mathrm{P}>0.05)$. MT-1E gene and mRNA expression levels in the Eca-109 and TE13 cell strains were significantly increased in the EX-H2598-M56-transfected group compared with the EX-NEG-M56-transfected group and the blank control group $(\mathrm{P}<$ $0.05)$. MT-1E gene and mRNA expressions did not differ significantly between the EX-NEGM56-transfected group and the blank control group $(\mathrm{P}>0.05)$ (Figure 2).
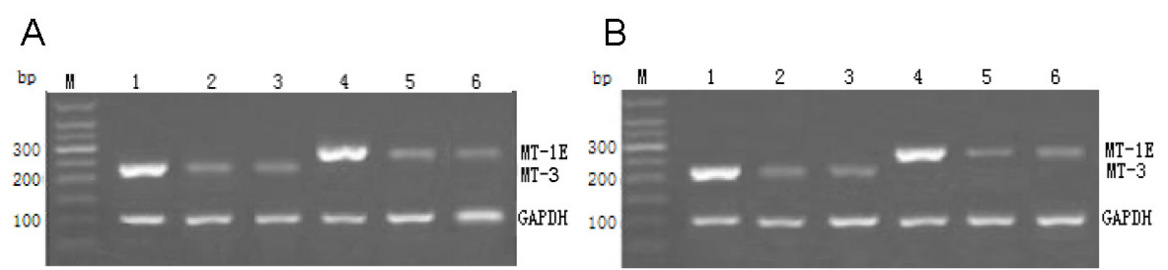

Figure 2. mRNA expression levels of MT-3 and MT-1E genes in transfected esophageal cancer cells after transfection for 48 h. A. Eca-109 cells. B. TE13 cells. Lane $M=$ molecular marker; Lane $1=$ EX-T3737-M03transfected group; lane 2 = EX-NEG-M03-transfected group; lane 3 = control group; lane 4 = EX-H2598-M56transfected group; lane 5 = EX-NEG-M56-transfected group; lane 6 = control group.

\section{Cell proliferation based on the MTT assay}

Results of the MTT assay showed that proliferation of esophageal cancer cells was significantly inhibited in the EX-T3737-M03-transfected group compared with the other groups $(\mathrm{P}<0.05)$. In Eca-109 cells, the cell proliferation inhibition rates were 22.45, 33.78, and $40.20 \%$ at 24,48 , and $72 \mathrm{~h}$, respectively. In TE13 cells, the 24,48 , and $72 \mathrm{~h}$ cell proliferation inhibition rates were $24.71,34.09$, and $39.68 \%$, respectively. No significant difference in cell proliferation was observed between the EX-H2598-M56- and EX-NEG-M56-transfected groups or the blank control group $(\mathrm{P}>0.05)$ (Figure 3).

A

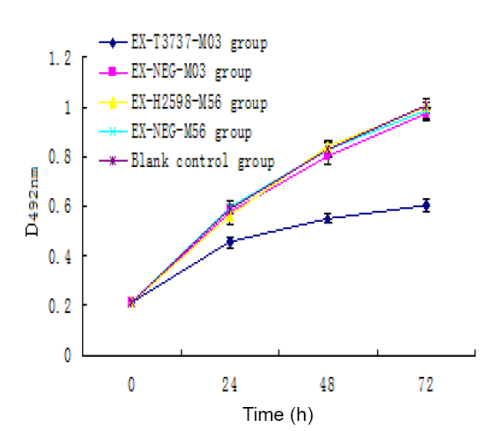

B

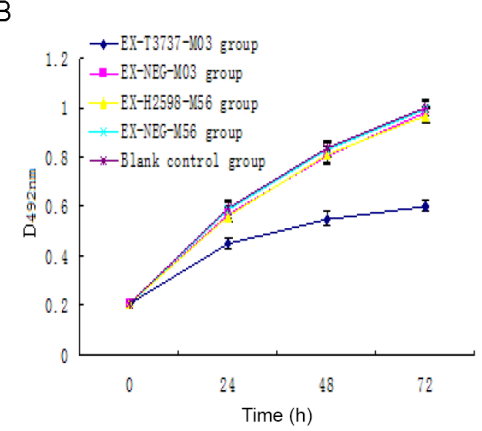

Figure 3. Proliferation of esophageal cancer cells after MT-3 and MT-1E genes transfection detected by the MTT method. A. Eca-109 cells. B. TE13 cells. 


\section{Effects of the MT-3 and MT-1E genes on the cell cycle and apoptosis of Eca-109 and TE13 cells}

The flow cytometry results showed that the proportion of cells in the G0/G1 phase increased significantly in the EX-T3737-M03-transfected group compared with the other groups $(\mathrm{P}<0.05)$. Furthermore, the proportion of cells in the $\mathrm{S}$ phase significantly reduced $(\mathrm{P}<0.05)$ and apoptosis significantly increased $(\mathrm{P}<0.05)$. The proportion of cells in other cell cycles and the apoptosis rates did not differ significantly between the EX-H2598-M56- and EXNEG-M56-transfected groups or the blank control group $(\mathrm{P}>0.05)$ (Figures 4 and 5, Table 1).
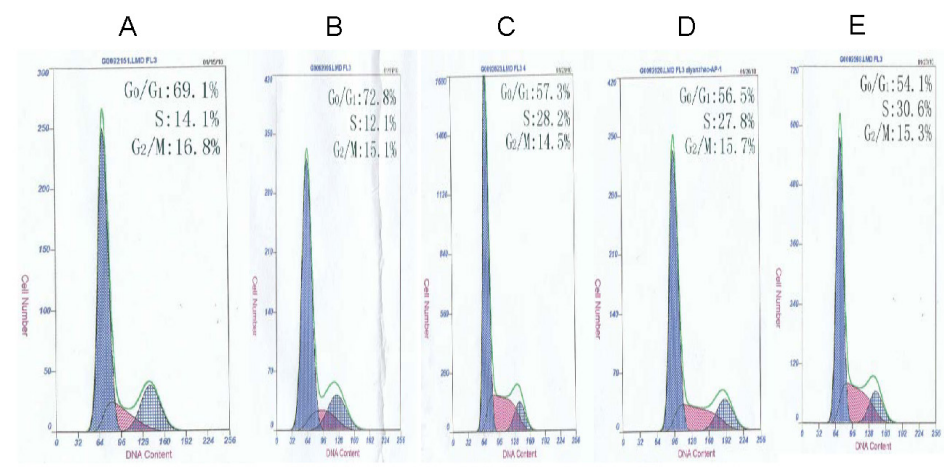

Figure 4. Cell cycle distributions of different esophageal cancer cell groups after transfection for $48 \mathrm{~h}$. A. Transfection with EX-T3737-M03 in Eca-109 cells. B. Transfection with EX-T3737-M03 in TE13 cells. C. Transfection with EX-H2598-M56 in Eca-109 cells. D. Transfection with EX-H2598-M56 in TE13 cells. E. Blank control.

A

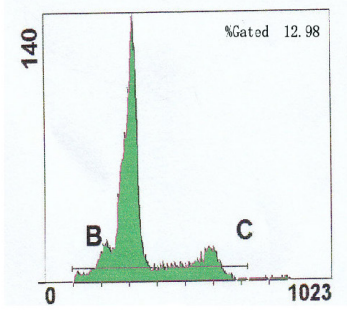

C

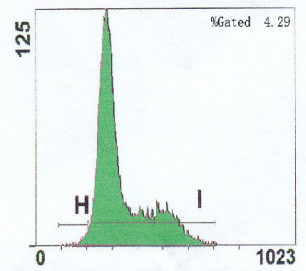

$\mathrm{D}$

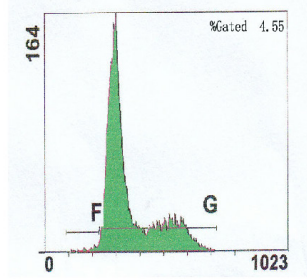

B

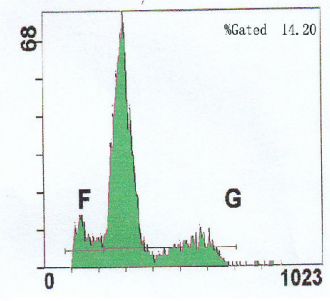

$E$

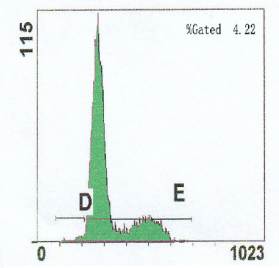

Figure 5. Changes of cell apoptosis of different esophageal cancer cell groups after transfection for $48 \mathrm{~h}$. A. Transfection with EX-T3737-M03 in Eca-109 cells. B. Transfection with EX-T3737-M03 in TE13 cells. C. Transfection with EXH2598-M56 in Eca-109 cells. D. Transfection with EX-H2598-M56 in TE13 cells. E. Blank control. 


Table 1. Effects of different transfected groups on cell cycle and apoptosis distribution of esophageal cancer
cell lines $(\%, \mathrm{~N}=6)$.
\begin{tabular}{lcccc}
\hline Group & $\mathrm{G}_{0} / \mathrm{G}_{1}$ & $\mathrm{~S}$ & $\mathrm{G}_{2} / \mathrm{M}$ & $14.72 \pm 1.08^{\Delta}$ \\
\hline EX-T3737-M03 & $70.97 \pm 1.58^{*}$ & $14.32 \pm 1.23^{*}$ & $14.07 \pm 1.10^{*}$ \\
EX- NEG-M03 & $53.20 \pm 2.13$ & $31.50 \pm 1.59$ & $15.30 \pm 1.38$ & $5.02 \pm 0.60$ \\
EX-H2598-M56 & $54.58 \pm 2.38^{+}$ & $30.15 \pm 1.77^{+}$ & $15.27 \pm 1.06^{+}$ & $4.69 \pm 0.72^{+}$ \\
EX- NEG-M56 & $53.02 \pm 2.16$ & $31.90 \pm 1.71$ & $15.08 \pm 0.78$ & $4.91 \pm 0.47$ \\
Blank control & $52.70 \pm 1.64$ & $31.45 \pm 1.02$ & $15.85 \pm 1.11$ & $4.74 \pm 0.49$ \\
\hline
\end{tabular}

Data are reported as means $\pm \mathrm{SD} . * \mathrm{P}<0.05, v s$ transfected groups and control group; ${ }^{\wedge \mathrm{P}}>0.05, v s$ transfected groups and control group; ${ }^{+} \mathrm{P}>0.05$, vs EX-NEG-M56 transfected group and control group.

\section{DISCUSSION}

MT proteins are a class of low molecular weight, cysteine-rich, small proteins that play important roles in the endogenous steady-state maintenance of metal ions, heavy metal detoxification, and free radicals clearance, among other functions (Miles et al., 2000) There are four MT isomers, which comprise 10 functional protein isoforms. In recent years, studies have shown that MT is highly expressed in many tumors, such as breast cancer, bladder cancer, testicular cancer, and thyroid cancer, among others (Cherian et al., 2003). MT was shown to be involved in tumor formation and has been closely associated with tumor cell differentiation, proliferation, and apoptosis.

Among the MT isoforms, the expressions of MT-1 and MT-2 have been most widely studied in esophageal cancer. Hishikawa et al. (1999) found that the high MT expression in esophageal cancer (mainly MT-1 and MT-2) was associated with lymph node metastasis, distant metastasis, and poor prognosis. Sun et al. (2006) also found that high expression levels of MT-1 and MT-2 were closely associated with the progress, low degree of differentiation, and lymph node metastasis of the esophageal disease.

The MT-3 gene was first discovered in 1991, and only one subtype has been identified to date with unique physiological functions. Zhou et al. (2003) found that MT-3 can perform active effects with at least one dozen active proteins. Additionally, abnormal expression of MT-3 was shown to be closely related with the occurrence and degree of malignant tumors such as prostate cancer (Garrett et al., 1999), bladder cancer (Sens et al., 2000), and breast cancer (Sens et al., 2001).

Cell proliferation and apoptosis are closely related in the process of tumor development. Many anti-cancer drugs have specific cell cycle blocking effects that induce apoptosis, which is often accompanied with growth inhibition. The cell cycle and apoptosis are closely related. In the normal cell cycle, two phases are the most important: the G1/S phase and the G2/M phase. At this time, the cells are going through complex molecular changes that are vulnerable to the influence of environmental conditions. Therefore, these two phases are the most frequently detected checkpoints in tumor cytology studies. In this study, flow cytometry was used to study the cell proportion and apoptosis rate at the G1/S and G2/M phases in Eca-109 and TE13 cells in order to clarify the effects of MT-3 and MT-1E transfection on tumor cell biological characteristics.

Our previous studies confirmed widespread MT-3 gene hypermethylation in esophageal carcinoma, which can lead to the downregulation of MT-3 expression levels (Tian et al., 2004; Sun et al., 2006). An immunohistochemistry study (Tian et al., 2008) revealed that MT-3 expression levels were significantly higher in esophageal cancer tissues compared with the cutting edge of normal tissue and benign esophageal lesions. Therefore, it may be speculated 
that the high MT-3 expression levels in esophageal cancer tissues result from carcinogenic factors, and the high expression of the MT-3 protein serves as a protective mechanism of the body by inhibiting cellular proliferation. Consequently, mutations of the MT-3 gene or CpG island hypermethylation would cause a decrease in its expression levels, and cancer cells would present high proliferative and poorly differentiated states.

In the present study, MT-3 and MT-1E gene transfection was performed in Eca-109 and TE13 cells using liposomal transfection technology. The RT-PCR results showed that MT-3 and MT-1E genes and mRNA were highly expressed, indicating that the transfection was successful with efficient expression. Cell proliferation and apoptosis were then detected to verify the hypothesis described above.

The MTT results showed that cell growth of the EX-T3737-M03-transfected group was significantly inhibited, whereas cell growth of the EX-H2598-M56-transfected group and other groups remained essentially unchanged. The flow cytometry results showed that at $48 \mathrm{~h}$ after transfection, the proportion of G0/G1 phase cells significantly increased in the EX-T3737-M03transfected group compared with the other groups. Meanwhile, the proportion of S phase cells decreased, and the apoptosis rates significantly increased. The proportion of cells in each cell cycle and the apoptosis rate in the EX-H2598-M56-transfected group did not show obvious changes compared with the EX-NEG-M56-transfected group and the blank control group.

These results suggested that the MT-3 gene might interfere with the G1/S phase of esophageal cancer cells to increase the proportion of G0/G1 phase cells. The proportion of cells in the S phase was significantly reduced, resulting in the G0/G1 arrest phenomenon. Therefore, cells in the DNA synthesis phase decreased and apoptosis was promoted, indicating that the MT-3 gene may inhibit the proliferation of esophageal cancer cells by changing the cell cycle to achieve antitumor effects.

Some studies have reported that MT-1 expression levels were positively correlated with the degree of malignancy of esophageal cancer (Sun et al., 2006). In the present study, proliferation effects were not apparent in the esophageal cancer cell strains Eca-109 and TE13 after MT-1E gene transfection.

There are two interpretations of this phenomenon. One is that because MT-1E is only one of at least six MT-1 subtypes, general changes in expression of MT-1 may not accurately be reflected by measuring changes in MT-1E. The other interpretation is that MT-1 might be only one of many downstream genes that are modified in the esophageal carcinogenesis process. Its influence had little effect on cell proliferation in the present study, which merits further investigation.

\section{ACKNOWLEDGMENTS}

Research supported by grants from the Hebei Science and Technology Research and Development Program (\#08276101D-9) and the Hebei Province Natural Science Foundation of China (\#C2011206066).

\section{REFERENCES}

Cherian MG, Jayasurya A and Bay BH (2003). Metallothioneins in human tumors and potential roles in carcinogenesis. Mutat. Res. 533: 201-209.

Garrett SH, Sens MA, Shukla D, Nestor S, et al. (1999). Metallothionein isoform 3 expression in the human prostate and cancer-derived cell lines. Prostate 41: 196-202. 
Guo Q and Tian ZQ (2010). Penetration effectiveness of air-to-ground, the different subtypes of metallothionein mRNA in esophageal squamous cell carcinoma. Pract. Oncol. 25: 519-522.

Hishikawa Y, Koji T, Dhar DK, Kinugasa S, et al. (1999). Metallothionein expression correlates with metastatic and proliferative potential in squamous cell carcinoma of the oesophagus. Br. J. Cancer 81: 712-720.

Miles AT, Hawksworth GM, Beattie JH and Rodilla V (2000). Induction, regulation, degradation, and biological significance of mammalian metallothioneins. Crit. Rev. Biochem. Mol. Biol. 35: 35-70.

Sens MA, Somji S, Lamm DL, Garrett SH, et al. (2000). Metallothionein isoform 3 as a potential biomarker for human bladder cancer. Environ. Health Perspect. 108: 413-418.

Sens MA, Somji S, Garrett SH, Beall CL, et al. (2001). Metallothionein isoform 3 overexpression is associated with breast cancers having a poor prognosis. Am. J. Pathol. 159: 21-26.

Smith E, Drew PA, Tian ZQ, De Young NJ, et al. (2005). Metallothionien 3 expression is frequently down-regulated in oesophageal squamous cell carcinoma by DNA methylation. Mol. Cancer 4: 42.

Sun XF, Du JJ and Meng L (2006). Metallothionein expression in esophageal squamous cell carcinoma and its relationship apoptosis and proliferation. Sin. Shandong Univ. 44: 384-388.

Tian ZQ and Liu JF (2004). Penetration effectiveness of air-to-ground, such as esophageal squamous cell carcinoma of the MT-3 gene CpG island methylation and its clinical significance. Pract. Oncol. 19: 386-389.

Tian ZQ, Wang XL and Wu W (2008). Metallothionein expression in esophageal carcinoma and its relationship with the expression of metallothionein 1 and 2. Cancer 27: 160-164.

Zhou B, Yang W, Ji JG and Bing-Gen RU (2003). Transiently transfected metallothionein-3 gene posterity neuroblastoma cell line SH-SY5Y cell proteins differentially expressed. Biochem. Biophys. 35: 522-528. 